\title{
Contribution of D-Dimer determination in the exclusion of deep venous thrombosis in spinal cord injury patients
}

\author{
J Roussi*,1, S Bentolila $^{1}$, L Boudaoud ${ }^{1}$, N Casadevall ${ }^{1}$, C Vallée ${ }^{2}$, R Carlier $^{2}$, S Lortat-Jacob ${ }^{3}$, O Dizien ${ }^{3}$ and \\ B Bussel ${ }^{3}$ \\ ${ }^{1}$ Laboratory of Hematology, Raymond Poincaré Teaching Hospital and Paris V University, France; ${ }^{2}$ Department of \\ Radiology, Raymond Poincaré Teaching Hospital and Paris V University, France; ${ }^{3}$ Department of Rehabilitation, \\ Raymond Poincaré Teaching Hospital and Paris V University, France
} Deep vein thrombosis (DVT) is a common complication of paraplegia despite prophylactic
anticoagulant therapy. The diagnosis relies primarily on ultrasonography or phlebography;
these investigations are difficult, expensive and can be time-consuming in paraplegic patients.
Study Design: To evaluate the usefulness of coagulation activation markers in excluding a
diagnosis of DVT, D-Dimers, thrombin-antithrombin complexes, prothrombin fragments
$\left(\mathrm{F}_{1+2}\right)$ and activated factor VIIa.

Objectives: To improve the diagnosis of deep venous thrombosis in paraplegic patients.

Setting: This collaborative work was done at Raymond Poincaré Hospital, Garches, France.

Methods: To evaluate the usefulness of coagulation activation markers in excluding a diagnosis of DVT, D-Dimers (D-Di), thrombin-antithrombin (TAT) complexes, prothrombin fragments $\left(\mathrm{F}_{1+2}\right)$ and activated factor VIIa (FVIIa), were determined in a prospective study of 67 consecutive patients with paraplegia or tetraplegia. Doppler ultrasonography and/or phlebography of the lower limbs and $\mathrm{D}$-Di, TAT, $\mathrm{F}_{1+2}$ level determination were systematically done in each patient at admission to our rehabilitation unit.

Results: Despite prophylactic low molecular weight heparin therapy, six of the 67 patients developed DVT diagnosed by radiologic explorations. D-Di levels measured by a reference ELISA (Asserachrom D-Di, Diagnostica Stago) or a new rapid automated turbidimetric test (STA-Liatest D-Di) were greater than $500 \mathrm{ng} / \mathrm{ml}$ in all DVT patients and in 40 non-DVT patients, of whom most had urinary tract infections, osteomas, or pressure sores. D-Di values were normal in only $21 / 67$ patients $(31 \%)$. The negative predictive value of D-Di in our study was $100 \%$ since all DVT patients had D-Di values greater than $500 \mathrm{ng} / \mathrm{ml}$. TAT and $\mathrm{F}_{1+2}$ levels were not correlated with D-Di levels but also had a negative predictive value of $100 \%$. Comparison of D-Di levels obtained using the two tests showed that results of the reference ELISA were closely correlated to those of the new rapid automated turbidimetric. TAT, $\mathrm{F}_{1+2}$, and factor VIIa are not useful for measuring hypercoagulability in paraplegic or tetraplegic patients since no rapid tests for determining these parameters are available.

Conclusion: D-Di levels determined using an ELISA or a new rapid automated turbidimetric test have a good negative predictive value for DVT in paraplegic or tetraplegic patients and may reduce the need for Doppler ultrasonography and/or phlebography by $31 \%$.

Keywords: D-Dimers; deep venous thrombosis; spinal cord injury

\section{Introduction}

Deep vein thrombosis (DVT) and pulmonary embolism (PE) remain common complications of spinal cord injury ${ }^{1}$ despite widespread use of prophylactic anticoagulant therapy. ${ }^{2,3}$ The clinical diagnosis of venous thrombosis is extremely difficult in such patients and the prevalence of thromboembolic complications has varied across studies, ${ }^{4,5}$ in particular as a result of differences in diagnostic methods; in the great majority

*Correspondence: Dr Jacqueline Roussi, Laboratoire d'hématologie, Hôpital Raymond Poincaré, 104 Boulevard Raymond Poincaré, Garches, 92380 France of the cases, radiologic explorations are necessary particularly ultrasonography and/or phlebography. Over the last few years, several markers for coagulation activation and fibrinolysis have been identified. Among them, plasma D-Dimer (D-Di) levels determined using an ELISA offer high sensitivity $(97 \%)$ and negative predictive value $(95 \%)$ for excluding venous thromboembolism in ambulatory patients and pulmonary embolism. ${ }^{6-9}$ Plasma D-Di is a cross-linked fibrin degradation product that reflects fibrin formation and subsequent dissolution. In a previous study, ${ }^{10}$ we evaluated haemostatic function, endothelial reactivity, 
and coagulation activation in patients with traumarelated paraplegia or tetraplegia. We found that prolonged bedrest was not associated with the recovery of fibrinolytic activity, and that D-Di levels were increased in $70 \%$ of DVT patients and in many non-DVT patients with conditions such as pressure sores, infections, or osteomas.

To our knowledge, the usefulness of D-Di levels for ruling out DVT in patients with paraplegia or tetraplegia has not yet been studied. Our goal was to determine the negative predictive value of $\mathrm{D}$-Di for DVT in 67 paraplegic or tetraplegic patients comparatively to Doppler ultrasonography and/or phlebography. We also measured three other markers for coagulation activation, namely thrombin antithrombin (TAT) complexes, prothrombin fragments $\left(\mathrm{F}_{1+2}\right)$ and activated factor VIIa. Because the reference ELISA for D-Di level determination is too timeconsuming to be useful in emergencies, we compared D-Di levels obtained using the classic ELISA (Asserachrom D-Di, Diagnostica Stago, Asnières, France) and a fast new D-Di assay (STA-Liatest DDi, Diagnostica Stago). We then determined if these two assays provided a greater than 95\% negative predictive value for DVT.

\section{Methods}

\section{Patients}

Sixty-seven consecutive patients with paraplegia or tetraplegia (52 males and 15 females) aged $17-72$ years were included in this prospective study. The 59 patients with paraplegia of $1-6$ months duration were receiving prophylactic low molecular weight heparin therapy, whereas the eight patients with longer paralysis durations were not. Written informed consent was obtained from each patient prior to study inclusion, and the study was conducted in accordance with the Declaration of Helsinki and with French legislation on biomedical research studies in humans. Doppler ultrasonography of the lower limbs and haemostasis tests including D-Di, $\mathrm{F}_{1+2}$ and FVIIa level determination were systematically done in each patient at admission to our rehabilitation unit. This screening was systematically performed for patients on hospital admission.

\section{Blood samples}

Venous blood was drawn directly into tubes containing EDTA $0.17 \mathrm{M}$ for blood cell counts, tubes containing sodium citrate $0.129 \mathrm{M}$ (1 vol/9 vol) (Vacutainer, Becton Dickinson, France) for haemostasis parameter determination, and Diatubes (Venoject) containing trisodium citrate $0.11 \mathrm{M}$, theophylline $15 \mathrm{nM}$, adenosine $37 \mathrm{nM}$, and dipyridamole $0.198 \mathrm{~nm}$ for TAT and $\mathrm{F}_{1+2}$ determination. Platelet-poor plasma obtained by blood centrifugation at $2700 \mathrm{~g}$ for $20 \mathrm{~min}$ at $15^{\circ} \mathrm{C}$ within $1 \mathrm{~h}$ of sampling was divided in $1 \mathrm{ml}$ aliquots and frozen at $-80^{\circ} \mathrm{C}$. Coagulation tests were performed within 1 month after blood sampling, except fibrinogen and D-Di determination, which were done on fresh platelet-poor plasma immediately after centrifugation.

\section{Imaging studies and blood tests}

Ultrasonography with pulsed color flow Doppler was performed at the time of blood sampling for the coagulation study, using the same ultrasound unit (Acuson XP 128) in all patients. The venous system was examined between the vena cava and the ankle veins. Enlargement, absence of compressibility, absence of a color Doppler signal, or direct visualisation of the thrombus were considered to indicate venous thrombosis. Patients whose veins were not completely visualised by Doppler ultrasonography underwent phlebography done using a conventional technique involving insertion of butterfly needles into the dorsal vein of each foot. A non-ionic contrast medium (Omnipaque-Nycomed Imaging Norway or Iopamiron-Schering Imaging Germany) was injected via a power injector. Tourniquets were used to improve opacification of the deep veins.

Blood cell and platelet counts were determined using an automated counter (MaxM, Coultronics, Margency, France). All clotting parameter determinations including factor VIIa (Staclot VIIa-rTF, Diagnostica Stago, France) were performed using a fully automated apparatus (STA, Diagnostica Stago, France). Prothrombin time, activated partial prothrombin time were respectively performed with Neoplastin CI and PTTA reagent (Diagnostica Stago). Fibrinogen was assayed using the Clauss method (Fibriprest A, Diagnostica Stago), with results in $\mathrm{mg} / \mathrm{dl}$. TAT and $\mathrm{F}_{1+2}$ were assayed using an ELISA (Enzygnost TAT and Enzygnost $\mathrm{F}_{1+2}$ respectively, from Dade-Behring, Paris la Défense, France) as recommended by the manufacturer.

Two commercially available D-Di kits were used, one classic ELISA, Asserachrom D-Di (Diagnostica Stago) and a new fast method, STA-Liatest D-Di (Diagnostica Stago). STA-Liatest D-Di is an automated turbidimetric test involving use of latex beads coated with two monoclonal D-Di antibodies that agglutinate in the presence of D-Di. The Asserachrom D-Di kit was used as the reference assay, in compliance with international recommendations. ${ }^{6,11}$

The radiologists were unaware of laboratory test results, and the laboratory technicians were unaware of imaging study findings. Statistical evaluation of the data involved Student's $t$-test done using the Statgraphics system (Uniware).

\section{Results}

Patient characteristics

Mean age was 34 years with a range of $17-72 ; 84 \%$ of patients were younger than 30 years. Over three- 
quarters of the patients were males (77\%). Eighty-eight per cent of patients were on prophylactic anticoagulant therapy, usually consisting in a low molecular weight heparin. Fifty patients had paraplegia and 17 tetraplegia, with injury being the most common cause. Six of the 67 patients $(9 \%)$ developed DVT diagnosed by Doppler ultrasonography; three out of these six patients underwent a phlebography which confirmed the diagnosis of DVT done by ultrasonography. All six were on prophylactic low molecular weight heparin therapy at the time of the DVT.

Platelet count were in the normal range $(304 \pm 140$ $\left.10^{9} / 1\right)$. The clotting parameters results were the following: (mean \pm standard deviation) prothrombin time $91 \pm 9 \%$, activated partial prothrombin time $35 \pm 4.5$ seconds, fibrinogen $4.3 \pm 1.5 \mathrm{mg} / \mathrm{dl}$, factor VIIa $43 \pm 22 \mathrm{mUI} / \mathrm{ml}$ (normal: $5-85 \mathrm{mUI} / \mathrm{ml}$ ).

\section{Markers for coagulation activation}

D-Di levels by the reference assay (Asserachrom D-Di) were below $500 \mathrm{ng} / \mathrm{ml}$ in 21 patients and increased $(>500 \mathrm{ng} / \mathrm{ml})$ in 46 , including six with DVT and 40 without DVT (Table 1). Many of the non-DVT patients had urinary tract infection, pressure sores, or osteoma. The negative predictive value of $\mathrm{D}$ - $\mathrm{Di}$ for DVT was $100 \%$.

TAT and $\mathrm{F}_{1+2}$ were normal in $60 \%$ of patients. No correlation was found between TAT and $\mathrm{F}_{1+2}$ levels (Figure 1). Neither were TAT or $\mathrm{F}_{1+2}$ levels correlated with D-Di levels. All the DVT patients had elevated levels of TAT, $\mathrm{F}_{1+2}$, and factor VIIa, yielding a negative predictive value for DVT of $100 \%$ for each of these three markers.

Using D-Di, TAT, $\mathrm{F}_{1+2}$, and VIIa in combination did not provide a useful increase in the positive predictive value, and retained a negative predictive value of $100 \%$.

Comparison of D-Di levels with the Asserachrom D-Di and Liatest D-Di kits

Table 2 shows the regression curve slopes and coefficients of correlation for the Liatest D-Di kit versus the reference ELISA. When D-Di levels were below $500 \mathrm{ng} / \mathrm{ml}$, levels by the Liatest D-Di were lower (slope 0.91) overall than those obtained using the Asserachrom D-Di kit. Results were discordant in three patients $(500,440$, and $310 \mathrm{ng} / \mathrm{ml}$, respectively, by the Asserachrom D-Di versus 760, 1060, and $540 \mathrm{ng} /$ $\mathrm{ml}$, respectively, by the Liatest $\mathrm{D}$-Di); none of whom had DVT. When the levels were greater than $500 \mathrm{ng}$ / $\mathrm{ml}$, levels by the Liatest D-Di were higher (slope 1.6) than levels by the Asserachrom D-Di.

\section{Discussion}

DVT and PE are complications of spinal cord injury that occur predominantly during the first $2-3$ months and are major causes of morbidity and mortality. ${ }^{12}$

The diagnostic value of $\mathrm{D}$-Di in thromboembolic disorders has been recognised for more than two decades. The negative predictive value of $\mathrm{D}$-Di for DVT has been studied by a number of investigators. ${ }^{11,13-15}$ In our previous study, ${ }^{10}$ we showed that $70 \%$ of paraplegic patients had high D-Di levels, and that D-Di levels were significantly increased not only in DVT but also in other conditions such as pressure sores, osteomas, and infections. Elevated D-Di levels in DVT suggest persistence of the thrombotic process without recovery of a normal fibrinolytic potential.

Table 1 D-Dimer levels obtained using the reference ELISA (Asserachrom D-Di) in 67 paraplegic or tetraplegic patients

\begin{tabular}{|c|c|c|}
\hline $\begin{array}{l}\mathrm{n}=67 \\
\text { patients }\end{array}$ & $\begin{array}{c}D-D i \text { ELISA } \\
<500 \mathrm{ng} / \mathrm{ml} \\
\text { (Asserachrom D-Di) }\end{array}$ & $\begin{array}{c}D-D i \text { ELISA } \\
>500 \mathrm{ng} / \mathrm{ml} \\
(\text { Asserachrom } D-D i)\end{array}$ \\
\hline $\begin{array}{l}\text { Deep } \\
\text { venous } \\
\text { thrombosis }\end{array}$ & $\begin{array}{c}0 \\
\text { (Negative predictive } \\
\text { value } 100 \% \text { ) }\end{array}$ & $\begin{array}{c}6 \\
(920-3000 \mathrm{ng} / \mathrm{ml})\end{array}$ \\
\hline $\begin{array}{l}\text { No deep } \\
\text { venous } \\
\text { thrombosis }\end{array}$ & 21 & $\begin{array}{c}40 \\
\text { (15 infections, } 10 \text { pressure } \\
\text { sores, } 3 \text { osteoma, } 12 \\
\text { unidentified causes) }\end{array}$ \\
\hline
\end{tabular}

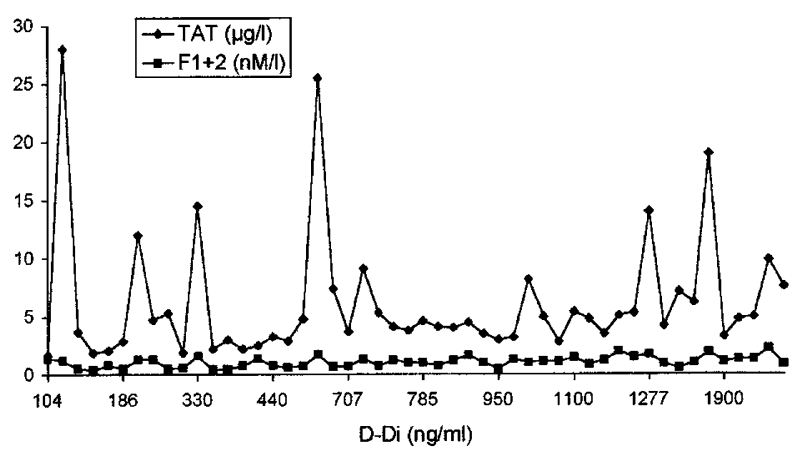

Figure 1 Comparison of TAT, $\mathrm{F}_{1+2}$, and D-Di results in 67 paraplegic or tetraplegic patients

Table 2 Correlation between D-Di results obtained using the reference test (Asserachrom D-Di) and the Liatest D-Di test

\begin{tabular}{lccc}
\hline $\begin{array}{l}\text { Asserachrom } \\
D-D i\end{array}$ & $\begin{array}{c}\text { STA liatest } D-D i \\
(<500 \mathrm{ng} / \mathrm{ml}) \mathrm{n}=22\end{array}$ & $\begin{array}{c}\text { STA liatest D-Di } \\
(>500 \mathrm{ng} / \mathrm{ml}) \mathrm{n}=45\end{array}$ & $\begin{array}{c}\text { STA liatest D-Di } \\
\text { Overall study group } \mathrm{n}=67\end{array}$ \\
\hline $\begin{array}{l}\text { Slope of the regression curve } \\
\text { Coefficient of correlation }\end{array}$ & 0.91 & 1.6 & 1.4 \\
& 0.55 & 0.94 & 0.95
\end{tabular}


Spinal cord injury is associated with several risk factors for DVT. In particular, slowing of the venous blood flow due to inflammation and absence of muscle contractions promote venous thrombosis and pulmonary embolism. ${ }^{16,17}$

The two aims of this study were to evaluate D-Di and other coagulation activation markers for excluding DVT in patients with paraplegia or tetraplegia and to determine if a rapid, quantitative, and automated $\mathrm{D}$-Di test that provides results within $1 \mathrm{~h}$ could be used. Rapid D-Di tests including sensitive latex assays and faster ELISAs or ELISA-derived assays have been developed to allow D-Di determination in emergencies. $^{18-20}$ STA-Liatest is a fast $(10 \mathrm{~min})$ automated turbidimetric test involving use of latex beads coated with two monoclonal D-Di antibodies that agglutinate in the presence of D-Di.

The negative predictive value of D-Di levels in our prospective study of 67 consecutive patients with paraplegia or tetraplegia was evaluated based on the results of a reference ELISA. D-Di levels were compared to findings from a Doppler ultrasonography and/or phlebography, as well as to levels of other coagulation activation markers, namely thrombin antithrombin (TAT), prothrombin fragments $\left(\mathrm{F}_{1+2}\right)$, and factor VIIa. Doppler ultrasonography has demonstrated good sensitivity and specificity versus phlebography for the detection of DVT. ${ }^{21,22}$

The reference ELISA (Asserachrom D-Di) was found to have a negative predictive value of $100 \%$, which is in keeping with earlier studies. ${ }^{8,9}$ Correlations were strong between the reference test and the STALiatest D-Di. In three patients, none of whom had DVT, a discrepancy was noted between the D-Di levels obtained using the reference Asserachrom D-Di test and the STA Liatest D-Di test. The slope of the regression curve between these two tests was smaller than one when D-Di levels were under $500 \mathrm{ng} / \mathrm{ml}$ but far larger than one when D-Di levels were greater than $500 \mathrm{ng} / \mathrm{ml}$. These findings suggest that a cut-off smaller than $500 \mathrm{ng} / \mathrm{ml}$ may be appropriate for the STALiatest test, and that studies in a larger number of patients are warranted to investigate this issue.

TAT, $F_{1+2}$ and FVIIa levels were not correlated with D-Di levels, but each had a negative predictive value of $100 \%$ for DVT. Using TAT, $F_{1+2}$, and/or VIIa alone or in combination failed to provide a useful increase in positive predictive value. Although TAT and $\mathrm{F}_{1+2}$ measure hypercoagulability in spinal cord injury patients, they cannot be used in the emergency diagnosis of thrombosis since no rapid assay is available for either marker.

In conclusion, D-Di is a useful marker for DVT in patients with paraplegia or tetraplegia. D-Di assay such as STA-Liatest D-Di is a fast and reliable test that can be used for ruling out DVT on an emergency basis. In our study $\mathrm{D}$-Di level determination had a negative predictive value for DVT of $100 \%$ and would have obviated the need for Doppler ultrasonography and/or phlebography in $31 \%$ of cases.

\section{Acknowledgements}

We are grateful to the technicians of the hematology laboratory for performing the assays needed for this study. This study was supported by a grant from the Fondation Garches. Presented at the XVIth congress of the International Society on Thrombosis and Haemostasis, July 1997.

\section{References}

1 Yelnik A et al. Systematic lower limb phlebography in acute spinal cord injury in 147 patients. Paraplegia 1991; 29: 253-260.

2 Yelnik A et al. Accidents thrombo-emboliques chez les paraplégiques: intérêt et limites du traitement anticoagulant preventif. Actualités en réeducation fonctionnelle et réadaptation, 11e série Masson (ed), 1986; 75-82, Paris.

3 Green D et al. Prevention of thromboembolism in spinal cord injury: role of low molecular weight heparin. Arch Phys Med Rehabil 1994; 75: $290-292$

4 Geerts WH et al. A prospective study of venous thromboembolism after major trauma. $N$ Eng J Med 1994; 331: 1601-1606.

$5 \mathrm{Kim}$ SW et al. Prevalence of deep venous thrombosis in patients with chronic spinal cord injury. Arch Phys Med Rehabil 1994; 75: $965-968$.

6 Bounameaux $\mathrm{H}$ et al. Measurement of plasma D. Dimer for diagnosis of deep venous thrombosis. Am J Clin Pathol 1989; 91: $82-85$.

7 Elias A et al. Assessment of D-Di measurement by ELISA or Latex methods in deep vein thrombosis diagnosed by ultrasonic duplex scanning. Fibrinolysis 1990; 4: 237-240.

8 Heijboer $\mathrm{H}$ et al. The use of the D-Dimer test in combination with non invasive testing versus serial non invasive testing alone for the diagnosis of deep vein thrombosis. Thromb Haemost 1992; 67: $510-513$.

9 Van Beek EJ et al. The role of plasma D-dimer concentration in the exclusion of pulmonary embolism. Br J Haematol 1996; 92: $725-732$

10 Boudaoud L et al. Endothelial fibrinolytic reactivity and the risk of deep venous thrombosis after spinal cord injury. Spinal Cord 1997; 35: $151-157$.

11 Bounameaux H, de Moerloose P, Perrier A, Reber G. Plasma measurement of D-Dimer as diagnostic aid in suspected venous thromboembolism: an overview. Thromb Haemost 1994; 71: 1 -6.

12 Gündüz $\mathrm{S}$ et al. Deep vein thrombosis in spinal cord injured patients. Paraplegia 1993; 31: 606-610.

13 Bounameaux $\mathrm{H}$ et al. Measurement of D-dimer in plasma as diagnostic aid in suspected pulmonary embolism. Lancet 1991 337: $196-200$

14 Raimondi $\mathrm{P}$ et al. D-dimer plasma concentration in various clinical conditions: Implication for the use of this test in the diagnostic approach of venous thromboembolism. Thromb Res 1993; 69: $125-130$.

15 Dale $\mathrm{S}$ et al. Comparison of three D-Dimer assays for the diagnosis of DVT: ELISA, Latex and Immunofiltration assay (Nycocard D-Dimer). Thromb Haemost 1994; 71: 270-274.

16 Myllynen P et al. The blood F. VIII : Ag/F VIII : C ratio as an early indicator of deep vein thrombosis during post-traumatic immobilization. J Trauma 1987; 27: 287-290.

17 Rossi EC et al. Sequential changes in factor VIII and platelets preceding deep vein thrombosis in patients with spinal cord injury. Br J Haematol 1980; 45: $143-151$.

18 Janssen MC et al. Reliability of five rapid D-Dimer assays compared to ELISA in the exclusion of deep venous thrombosis. Thromb Haemost 1997; 77: $262-266$.

19 Scarano L et al. Accuracy of two newly described D-Dimer tests in patients with suspected deep vein thrombosis. Thromb Res 1997; 86: $93-99$ 
20 Elias A et al. D-Dimer test and diagnosis of deep vein thrombosis: a comparative study of 7 assays. Thromb Haemost 1996; 76: $518-522$.

21 Elias A et al. Value of real time B mode ultrasound of deep vein thrombosis of the lower limbs. Int Angiol 1987; 6: 175-182.
22 Lensing AW, Prandoni P, Brandjes D. Detection of deep vein thrombosis by real time B mode ultrasonography. $N$ Engl J Med 1989; 320: $342-345$. 